\title{
Study on the Effect of Heat-Input on the Thickness of the Heat-Affected Zone of the SUS316L Steel Welded Joint by Numerical Simulation
}

Nghiên cứu ảnh hưởng của năng lượng đường đến chiều dày của vùng ảnh hưởng nhiệt liên kết giáp mối thép SUS316L bằng mô phỏng số

\author{
Vu Dinh Toai ${ }^{1 *}$, Do Van Long ${ }^{2}$ \\ ${ }^{1}$ Hanoi University of Science and Technology, Hanoi, Vietnam \\ ${ }^{2}$ The Central College of Transport No.2, Hai Phong, Vietnam \\ Email: toai.vudinh@hust.edu.vn
}

\begin{abstract}
The thickness of the heat-affected zone (HAZ) has a great influence on the strength of the welded joint, so one of the important tasks is to control the HAZ to a small enough level, through using the suitable heat-input $\left(q_{d}\right)$. In this study, the authors use SYSWELD software to compute and build a relationship between the heatinput and the thickness of the heat-affected zone in the plate thickness direction to find the right heat-input for researched welding joint. The simulation results show that when welding the root pass with $q_{d}>552 \mathrm{~J} / \mathrm{mm}$ and the cap pass with $754 \mathrm{~J} / \mathrm{mm}<q_{d}<1066 \mathrm{~J} / \mathrm{mm}$, the thickness of HAZ were increased with a function almost linearly.
\end{abstract}

Keywords: Heat-affected zone, HAZ, heat input, SUS316L steel, Sysweld.

Tóm tắt

Chiều dày của vùng ảnh hưởng nhiệt (HAZ) có ảnh hưởng lớn đến độ bền của liên kết hàn, do đó khi hàn nóng chảy thì một trong những nhiệm vụ quan trọng là phải khống chế vùng HAZ ở mức đủ nhỏ, thông qua việc sử dụng năng lượng đường $\left(q_{d}\right)$ phù hợp. Trong nghiên cứu này, nhóm tác giả sử dụng phần mềm SYSWELD để tính toán và xây dựng mối quan hệ giữa năng lượng đường với chiều dày của vùng ảnh hưởng nhiệt theo hướng chiều dày tấm để thông qua đó tìm ra năng lượng đường phù hợp với liên kết hàn nghiên cứu. Kết quả mô phỏng cho biết rằng khi hàn lớp lót với $q_{đ}>552 \mathrm{~J} / \mathrm{mm}$ và lớp phủ với $754 \mathrm{~J} / \mathrm{mm}<q_{đ}<1066 \mathrm{~J} / \mathrm{mm}$ thì chiều dày vùng HAZ tăng theo hàm gần như tuyến tính.

Từ khóa: Vùng ảnh hưởng nhiệt, HAZ, năng lượng đường, thép SUS316L, Sysweld

\section{Giới thiệu}

Khi hàn nóng chảy kim loại thì trong liên kết hàn luôn luôn tồn tại một vùng ảnh hưởng nhiệt (HAZ) và nó là nơi có độ bền thấp nhất của liên kết [1,2]. Đối với những vật liệu có chuyển biến thù hình khi nung nóng và làm nguội thì ở vùng ảnh hưởng nhiệt này sẽ diễn ra đồng thời rất nhiều quá trình, như: khuếch tán, chuyển biến pha, kết tinh lại, quá nhiệt, biến đổi cỡ hạt,... Còn ngay cả đối với vật liệu không có chuyển biến pha thì vùng ảnh hưởng nhiệt cũng luôn xảy ra hiện tượng thay đổi về cỡ hạt như mô tả trên hình 1 [1], làm cho tính chất của vật liệu bị thay đổi. Vùng HAZ liền kề với mối hàn sẽ có hạt thô do được cấp nhiều nhiệt từ mối hàn và có độ bền suy giảm, trong khi đó vùng $\mathrm{HAZ}$ gần với kim loại cơ bản sẽ có hạt mịn do được nguội nhanh và có độ bền, độ cứng tăng lên. Chênh lệch về độ bền, độ cứng của 2 vùng liền kề nhau này luôn sinh ra một ứng suất gọi là ứng suất biến đổi cấu trúc. Úng suất này cộng tác dụng với ứng suất do nung nóng cục bộ và ứng suất do chuyển biến pha,... sẽ hình thành lên một trường ứng suất dư sau hàn, làm giảm khả năng chịu tải của liên kết. Liên kết hàn nào có vùng $\mathrm{HAZ}$ càng lớn thì độ bền càng suy giảm.

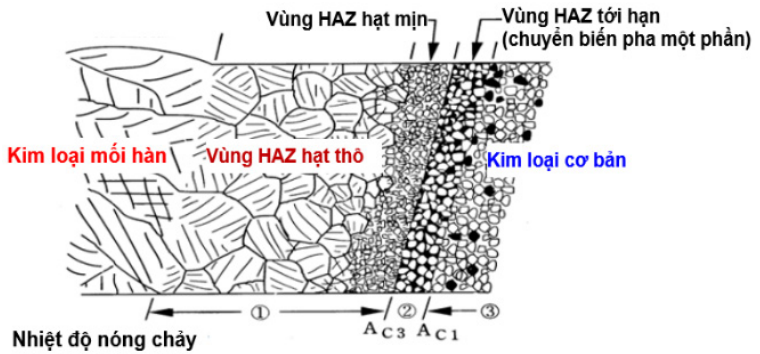

Hình 1. Cấu trúc vùng ảnh hưởng nhiệt của liên kết hàn nóng chảy

Cũng theo các tài liệu [1-3], chiều dày của vùng $\mathrm{HAZ}$ phụ thuộc bởi chế độ hàn đã sử dụng, mà trực tiếp là năng lượng đường $\left(q_{d}\right)$ cấp vào vùng hàn. Nếu $q_{d}$ cấp vào ít thì chiều dày vùng HAZ sẽ nhỏ nhưng nếu $q_{\text {d }}$ bé hơn mức cần thiết thì liên kết hàn sẽ không ngấu. Ngược lại nếu $q_{d}$ mà lớn quá mức cần thiết thì vùng HAZ sẽ quá lớn, làm giảm độ bền và khả năng làm việc

ISSN 2734-9381

https://doi.org/10.51316/jst.154.etsd.2021.31.5.10

Received: September 20, 2020; accepted: March 11, 2021 
của liên kết. Do vậy, việc xác định được quan hệ giữa

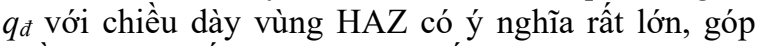
phần tìm ra chế độ hàn hợp lý đối với từng vật liệu và liên kết hàn cụ thể.

\section{Mô hình mô phỏng liên kết hàn giáp mối 2 lớp}

\subsection{Mô hình phần tử hữu hạn}

Mô hình nghiên cứu trong bài báo này là liên kết hàn giáp mối bằng thép không gỉ chịu nhiệt SUS316L với chiều dày $8 \mathrm{~mm}$ và được thực hiện bằng quá trình hàn MIG. Theo quy phạm AWS D1.6 [4] thì mỗi tấm phôi phải có kích thước tối thiểu là $150 \times 350 \times 8$ (dài $x$ rộng $x$ dày $[\mathrm{mm}]$ ). Cũng theo AWS D1.6 thì liên kết hàn giáp mối thép không gỉ dày $8 \mathrm{~mm}$ cần được vát mép dạng chữ $\mathrm{V}$ với các thông số cụ thể như sau: khe đáy $k=2 \mathrm{~mm}$, mặt đáy $d=2 \mathrm{~mm}$, góc rãnh hàn $\alpha=60^{\circ}$. Áp dụng các công thức trong tài liệu [5] ta xác định được số lớp hàn của liên kết hàn này là $n=2$. Sử dụng mô đun Visual-Mesh của phần mềm SYSWELD để xây dựng mô hình $3 \mathrm{D}$ và chọn loại phần tử 6 mặt 18 nút để chia lưới ta được mô hình PTHH như biểu diễn trên hình 2, gồm 24846 nút và 20500 phần tử 3D.
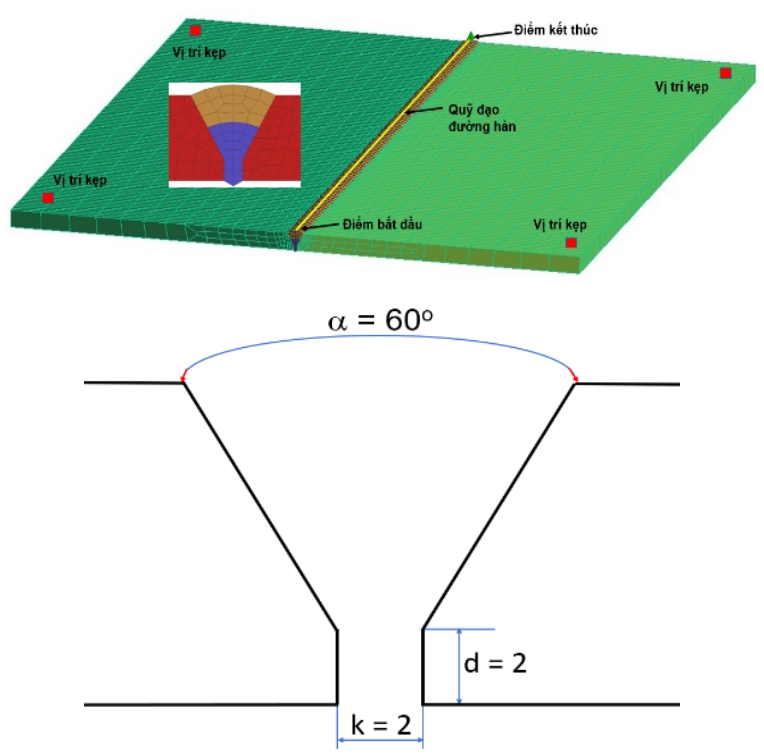

Hình 2. Mô hình PTHH, vị trí kẹp phôi và quỹ đạo đường hàn của liên kết giáp mối 2 lớp

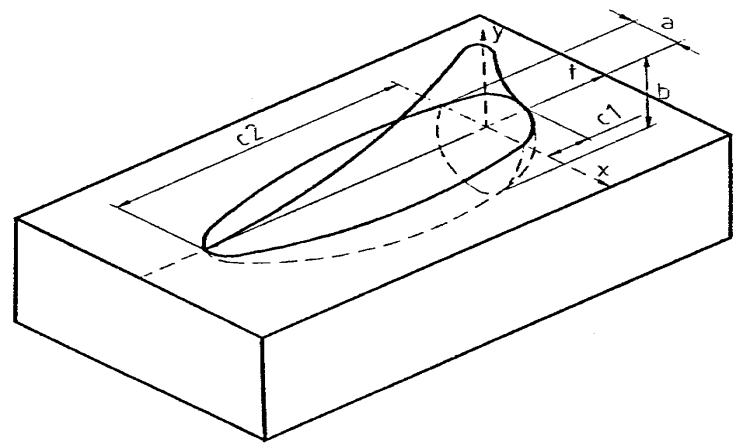

Hình 3. Mô hình nguồn nhiệt hồ quang di động của Radaj

\subsection{Mô hình nguồn nhiệt hàn}

Đối với các nguồn nhiệt hồ quang di động nói chung, nguồn nhiệt hàn MIG di động nói riêng thì mô hình nguồn nhiệt Ellipsoid của tác giả Radaj [6] như biểu diễn trên hình 3 thường được áp dụng để tính toán mô phỏng quá trình hàn bởi nó có độ chính xác cao. Mô hình nguồn nhiệt này đã được tập đoàn ESI tích hợp sẵn trong mô đun Visual-Weld của phần mềm SYSWELD.

Trong nghiên cứu này, tác giả sử dụng các hệ số tụ nhiệt phía trước hồ quang $f$, hệ số tản nhiệt phía sau hồ quang $r$, kích thước của vùng được phủ hồ quang $a$, $b, c 1, c 2$ cho các lớp hàn lót và hàn phủ như trình bày trong bảng 1 .

Bảng 1. Các thông số nguồn nhiệt hàn sử dụng

\begin{tabular}{|c|c|c|c|}
\hline Thông số & Giá trị & Thông số & Giá trị \\
\hline$q_{f}$ & $1,2 q_{d}$ & $q_{r}$ & $0,6 q_{\text {d }}$ \\
\hline$a$ (lót) & $4 \mathrm{~mm}$ & $b$ (lót) & $2 \mathrm{~mm}$ \\
\hline$c 1$ (lót) & $2 \mathrm{~mm}$ & $c 2$ (lót) & $6 \mathrm{~mm}$ \\
\hline$a$ (phủ) & $6 \mathrm{~mm}$ & $b$ (phủ) & $4 \mathrm{~mm}$ \\
\hline$c 1$ (phủ) & $3 \mathrm{~mm}$ & $c 2$ (phủ) & $9 \mathrm{~mm}$ \\
\hline
\end{tabular}

\subsection{Các thông số vật liệu}

Một bộ thông số đầy đủ về cơ - nhiệt - kim loại học (là hàm số của cả nhiệt độ và tổ chức kim loại) của thép không gỉ chịu nhiệt SUS316L, được xác định bằng lý thuyết nhiệt động - kim loại học và đo đạc bằng thực nghiệm, được tích hợp sẵn trong ngân hàng dữ liệu vật liệu trong phần mềm SYSWELD. Trong nghiên cứu này tác giả sử dụng bộ thông số cơ - nhiệt - kim loại học của thép SUS316L thể hiện từ hình 4 đến hình 9 [7].

\section{4. Điều kiện biên và điều kiện đầu}

Vỏ hay mặt bao phía ngoài của mô hình, nơi diễn ra các quá trình đối lưu và bức xạ nhiệt từ mô hình ra môi trường xung quanh là 26474 phần tử mặt $2 \mathrm{D}$ (hình 10 ) được xây dựng và gắn vào các phần tử $3 \mathrm{D}$ trên hình 2.

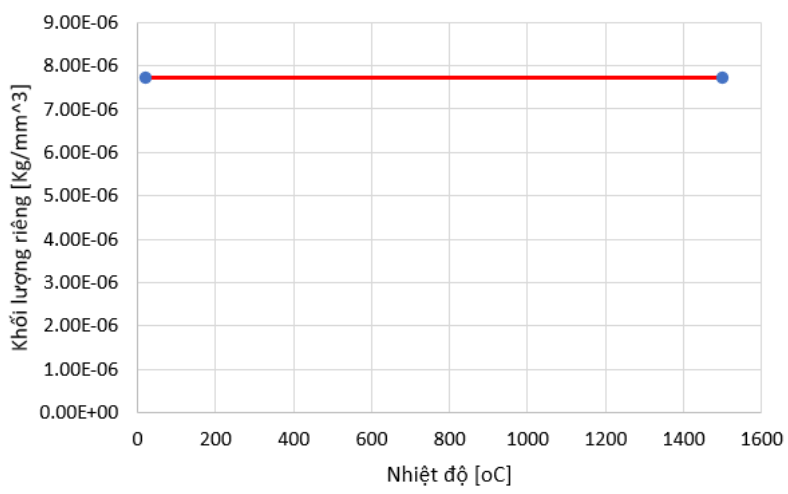

Hình 4. Khối lượng riêng của thép SUS316L 


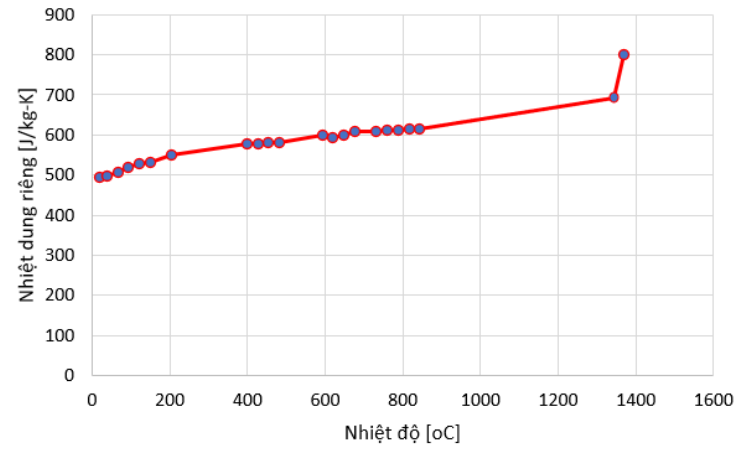

Hình 5. Nhiệt dung riêng của thép SUS316L

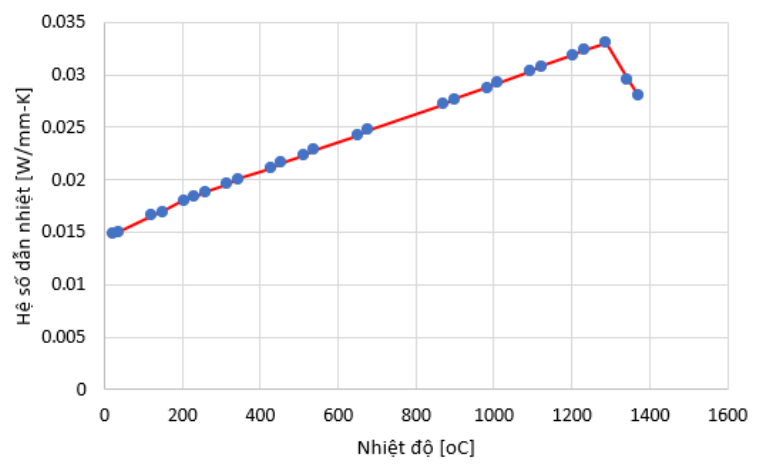

Hình 6. Hệ số dẫn nhiệt của thép SUS316L

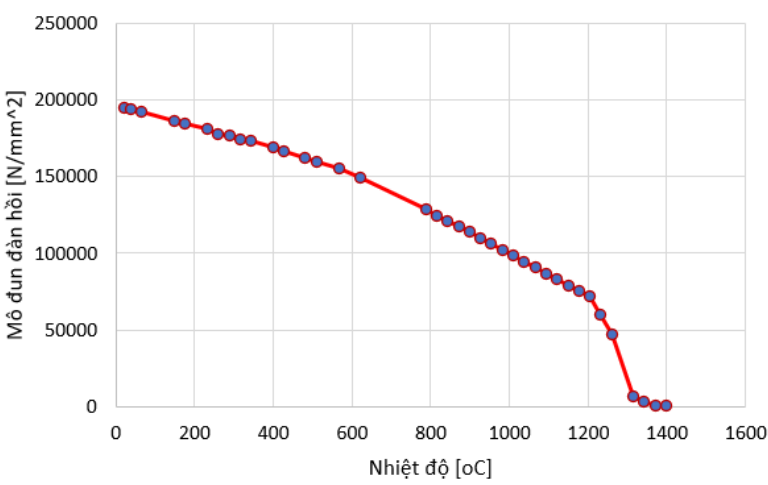

Hình 7. Mô đun đàn hồi của thép SUS316L

Hàm truyền nhiệt đối lưu và bức xạ này được xác định bằng thực nghiệm và được nhập vào phần mềm SYSWELD để tính toán. Trong nghiên cứu này tác giả sử dụng hàm truyền nhiệt trong điều kiện không khí tĩnh như mô tả trên hình 11 để tính toán.

Vị trí và kiểu gá kẹp trên mô hình yêu cầu phải được mô tả đúng với thực tế. Trong nghiên cứu này, mô hình được kẹp chặt ở 4 góc như thể hiện trên hình 2. Nhiệt độ ban đầu của toàn bộ mô hình lấy bằng $30^{\circ} \mathrm{C}$ (bằng nhiệt độ của môi trường không khí).

\subsection{Thiết lập các thông số tính toán}

Để bảo đảm độ chính xác thì các thông số mô phỏng phải đúng với thực tế và trong nghiên cứu này tác giả đã xây dựng 2 quỹ đạo đường hàn thẳng (không dao động ngang) theo đúng yêu cầu của hàn thép không gỉ $[2,3]$. Vị trí bắt đầu hồ quang của cả 2 lớp hàn

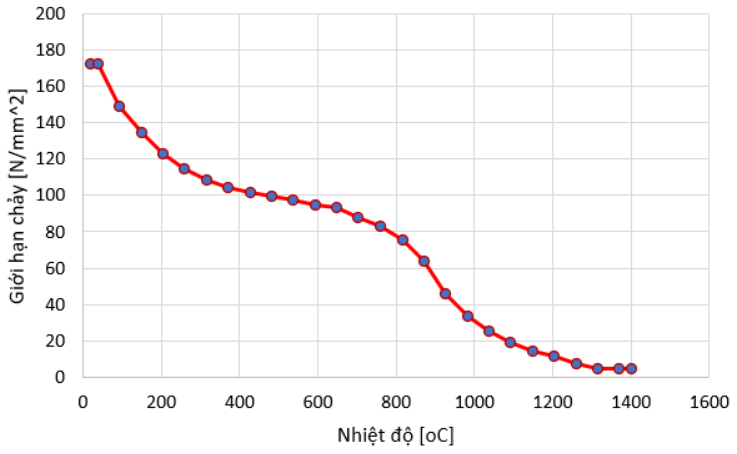

Hình 8. Giới hạn chảy của thép SUS316L

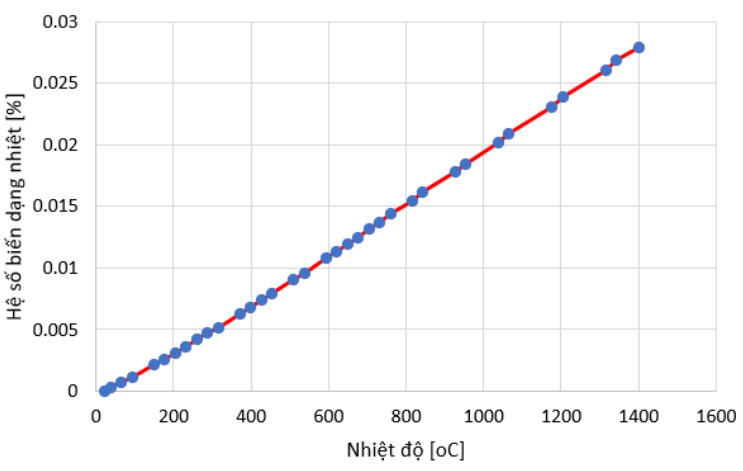

Hình 9. Hệ số biến dạng nhiệt của thép SUS316L

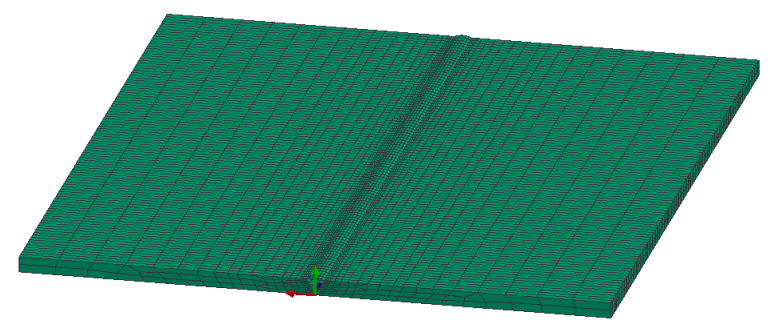

Hình 10. Các phần tử 2D là vỏ của mô hình

cùng nằm ở một đầu của mép hàn và điểm kết thúc hồ quang cũng cùng nằm ở cuối mép hàn như trình bày trên hình 2 .

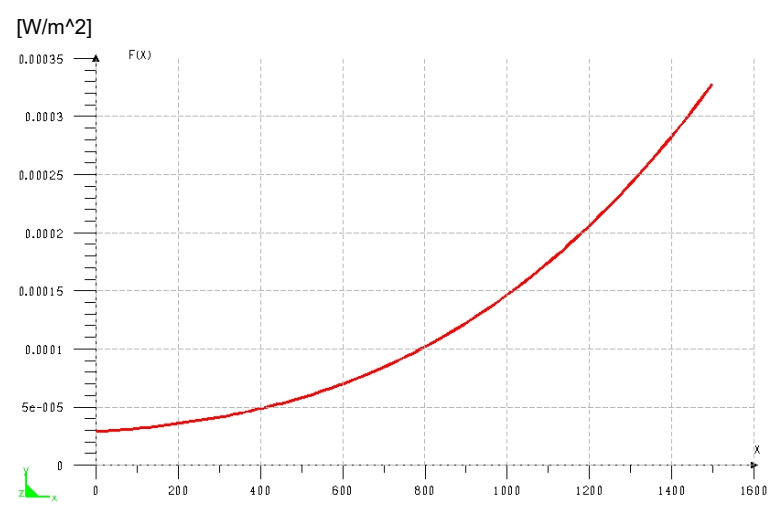

Hình 11. Hàm truyền nhiệt đối lưu và bức xạ ra môi trường không khí tĩnh 
Để nghiên cứu ảnh hưởng của năng lượng đường đến chiều dày vùng ảnh hưởng nhiệt của liên kết hàn trên hình 2 , các tác giả tiến hành tính toán ở nhiều dự án với các giá trị năng lượng đường khác nhau như mô tả trong bảng 2 . Xuất phát từ năng lượng đường sơ bộ tính theo lý thuyết (ở DA4), tiến hành mô phỏng lần lượt với các năng lượng đường giảm dần cho đến khi không đủ ngấu liên kết nữa thì dừng lại (DA1). Tiếp tục thực hiện tương tự theo hướng tăng năng lượng đường cho đến khi liên kết hàn bị cháy thủng thì dừng lại (DA6).

Bảng 2. Các thông số tính toán mô phỏng

\begin{tabular}{|c|c|c|}
\hline Dự án & $\begin{array}{c}\text { Năng luợng đưòng } \\
\text { lớp lót qđi }[\mathrm{J} / \mathrm{mm}]\end{array}$ & $\begin{array}{c}\text { Năng lương } \\
\text { đương lóp phủ } \\
q_{\text {đap }}[\mathrm{J} / \mathrm{mm}]\end{array}$ \\
\hline DA1 & 440 & 702 \\
\hline DA2 & 506 & 754 \\
\hline DA3 & 552 & 910 \\
\hline DA4 & 600 & 1066 \\
\hline DA5 & 650 & 1118 \\
\hline DA6 & 702 & 1170 \\
\hline
\end{tabular}

Bảng 3. Chiều dày của vùng $\mathrm{AHN}$ liên kết hàn

\begin{tabular}{|c|c|c|c|}
\hline Dư án & $H A Z_{1}[\mathrm{~mm}]$ & $H A Z_{2}[\mathrm{~mm}]$ & $H A Z_{3}[\mathrm{~mm}]$ \\
\hline DA1 & 5,13096 & 3,92104 & 2,86588 \\
\hline DA2 & 5,39199 & 4,34354 & 3,20565 \\
\hline DA3 & 5,89471 & 4,79153 & 3,42982 \\
\hline DA4 & 6,24158 & 5,29374 & 3,7287 \\
\hline DA5 & 6,58563 & 5,661 & 4,28278 \\
\hline DA6 & 6,94439 & 5,86705 & 4,64728 \\
\hline
\end{tabular}

\section{Kết quả nghiên cứu và bàn luận}

Sau khi xây dựng mô hình khối, chia lưới phần tử $3 \mathrm{D}$, tạo vỏ phần tử 2D trao đổi nhiệt, đặt điều kiện gá kẹp, tạo các đường hàn, gán các thuộc tính vật liệu, gán mô hình nguồn nhiệt và đặt tải lên mô hình là các năng lượng đường trong bảng 2 rồi tiến hành tính toán ta thu được các kết quả dưới đây.

Theo tài liệu [8], do nhiệt độ nóng chảy của thép SUS316L là $1450{ }^{\circ} \mathrm{C}$ và nhiệt độ bắt đầu chuyển biến pha của thép này là $800^{\circ} \mathrm{C}$ nên nhóm tác giả điều chỉnh thang đo để biểu diễn các đường đẳng nhiệt có chứa 2 giá trị quan trọng là $1450{ }^{\circ} \mathrm{C}$ (màu đỏ) và $800^{\circ} \mathrm{C}$ (màu cam). Sử dụng chức năng hiển thị trạng thái nhiệt độ tổng hợp lớn nhất và công cụ đo đạc khoảng cách giữa các điểm hoặc nút trong mô đun Visual-Viewer của phần mềm SYSWELD ta sẽ xác định được chiều dày của vùng ảnh hưởng nhiệt tại bất kỳ vị trí nào trong liên kết hàn. Để đánh giá khả năng ngấu của các mép hàn cũng như khả năng ngấu giữa các đường hàn với nhau, tác giả sử dụng chức năng hiện đường bao giữa các đối tượng bằng đường màu trắng như thể hiện trên hình 13.

Ký hiệu chiều dày của vùng ảnh hưởng nhiệt tại đáy liên kết hàn là $H A Z_{1}$, tại vị trí giữa chiều dày của liên kết là $\mathrm{HAZ}_{2}$ và ở trên bề mặt của liên kết là $\mathrm{HAZ}_{3}$, ta thu được các kết quả như Hình 12.

Kết quả tính toán trường nhiệt độ tổng hợp phân bố trên tiêt diện ngang của liên kết hàn khi hàn với năng lượng đường $440 \mathrm{~J} / \mathrm{mm}$ đối với lớp lót và $702 \mathrm{~J} / \mathrm{mm}$ đối với lớp phủ (dự án DA1) được thể hiện trên hình $12 \mathrm{a}$. Trong trường hợp này ta thấy liên kết hàn chưa ngấu đáy, vùng ảnh hưởng nhiệt tại 3 vị trí xem xét là $\mathrm{HAZ}_{1}=5,13096 \mathrm{~mm}, \mathrm{HAZ}_{2}=3,92104 \mathrm{~mm}$ và $\mathrm{HAZ}_{3}=2,86588 \mathrm{~mm}$. Kết quả mô phỏng cho thấy $\mathrm{HAZ}_{1}>\mathrm{HAZ}_{2}>\mathrm{HAZ}_{3}$, nghĩa là chiều dày của vùng ảnh hưởng nhiệt giảm dần từ đáy lên trên bề mặt liên kết.

Khi hàn với chế độ hàn có $q_{\text {dl }}=506 \mathrm{~J} / \mathrm{mm}$ và $q_{đ p}=754 \mathrm{~J} / \mathrm{mm}$ (DA2) ta thu được kết quả thể hiện trên hình $12 \mathrm{~b}$. Ở chế độ hàn này thì liên kết hàn ngấu rất đều: mức độ ngấu ở đáy, ở giữa và ở mặt liên kết là tương đương nhau và bề dày của vùng ảnh hưởng nhiệt đo được lần lượt là $\mathrm{HAZ}_{1}=5,39199 \mathrm{~mm}$, $\mathrm{HAZ}_{2}=4,34354 \mathrm{~mm}, \mathrm{HAZ}_{3}=3,20565 \mathrm{~mm}$. Ta thấy rằng bề dày vùng ảnh hưởng nhiệt cũng giảm dần từ đáy lên trên mặt liên kết, tuy nhiên so với DA1 thì ở DA2 mức độ giảm đều đặn hơn.

Khi tăng năng lượng đường lớp lót lên $552 \mathrm{~J} / \mathrm{mm}$ và lớp phủ lên $910 \mathrm{~J} / \mathrm{mm}$ (DA3) ta thu được kết quả trên hình $12 \mathrm{c}$. Trường hợp này liên kết hàn cũng ngấu hoàn toàn và khá đều như DA2, nhưng chiều dày của vùng ảnh hưởng nhiệt ở cả 3 vị trí xem xét đều lớn hơn so với DA2.

Nếu như liên kết được hàn với $q_{\text {dl }}=600 \mathrm{~J} / \mathrm{mm}$ và $q_{d p}=1066 \mathrm{~J} / \mathrm{mm}$ (DA4) thì ta thu được kết quả trên hình $12 \mathrm{~d}$. Với chế độ hàn này thì liên kết hàn cũng ngấu hoàn toàn, tuy nhiên độ ngấu không còn đều đặn như các dự án $\mathrm{DA} 2$ và $\mathrm{DA} 3$ nữa. Bề dày vùng ảnh hưởng nhiệt trong trường hợp này cũng lớn hơn các trường hợp trước và lần lượt là $\mathrm{HAZ}_{1}=6,24158 \mathrm{~mm}$, $\mathrm{HAZ}_{2}=5,29374 \mathrm{~mm}, \mathrm{HAZ}_{3}=3,7287 \mathrm{~mm}$. 


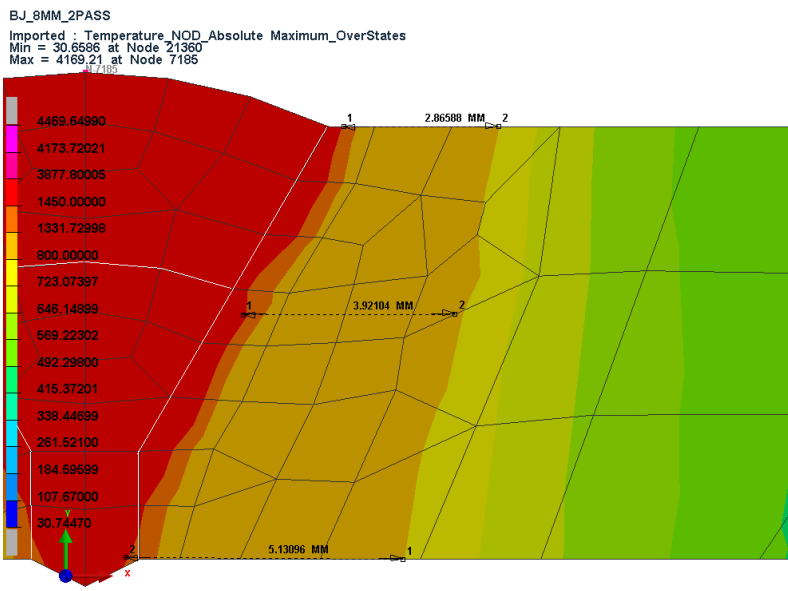

a) DA1

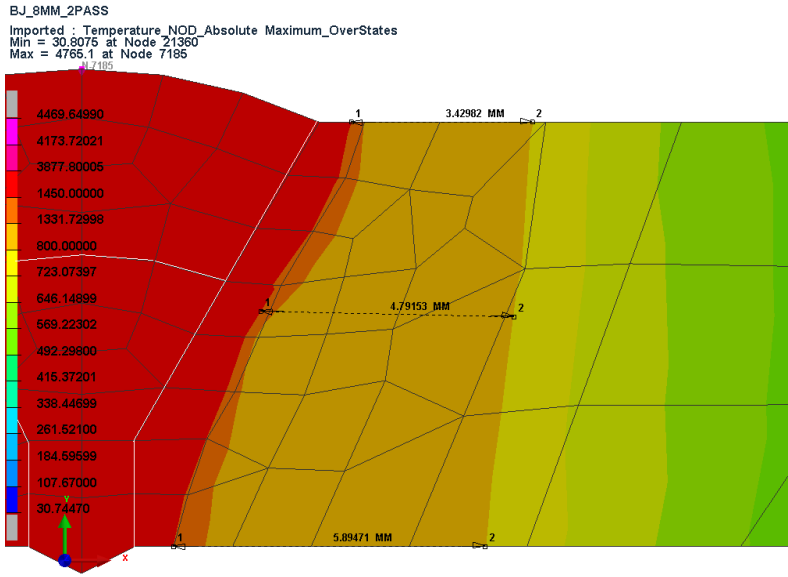

c) DA3

BJ_BMM_2PASS

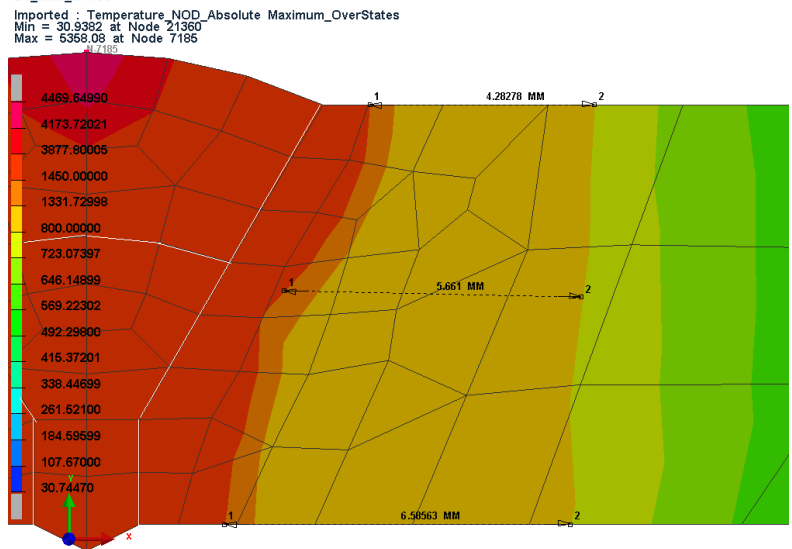

e) DA5

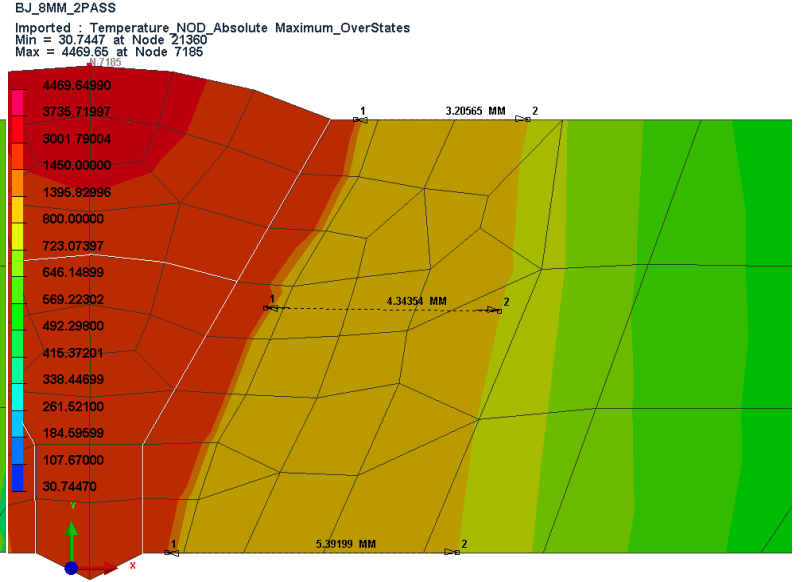

b) DA2

BJ_8MM_2PASS

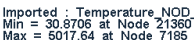

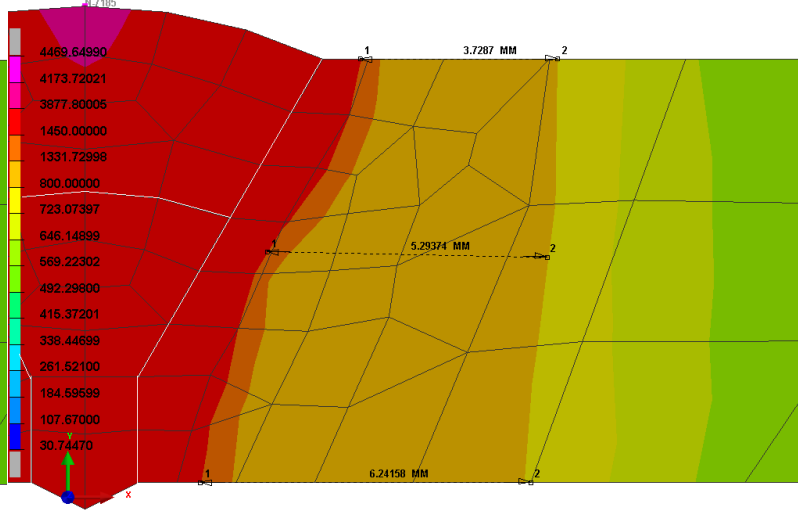

d) DA4

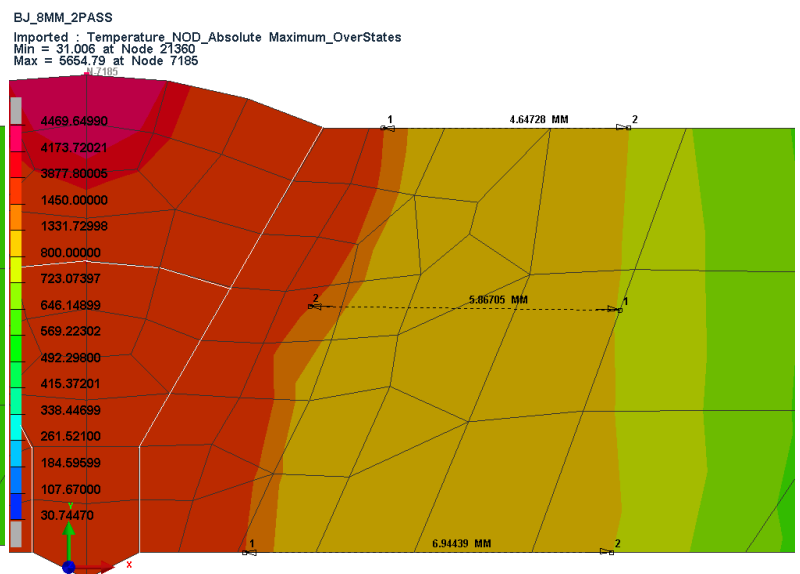

f) DA6

Hình 12. Phân bố nhiệt độ cực đại trên tiết diện ngang của liên kết hàn và bề rộng vùng ảnh hưởng nhiệt.

Tiếp tục tăng năng lượng đường như ở các DA5 và DA6 (bảng 2) ta thu được các kêt quả tương ứng trên hình $12 \mathrm{e}$ và hình $12 \mathrm{f}$. Hai trường hợp này cũng giống như DA4: chiều sâu ngấu không đều, khả năng dẫn đến khuyết tật chảy xệ và cháy cạnh liên kết hàn do chiều sâu chảy ở cả đáy và mặt đều lớn. Bề dày của vùng ảnh hưởng nhiệt ở cả 3 vị trí xem xét đều lớn (bảng 3) sẽ là nguyên nhân làm suy giảm độ bền của liên kết hàn nếu hàn ở các chế độ này.
Đồ thị trên hình 13 biểu diễn ảnh hưởng của năng lượng đường đến chiều dày của vùng ảnh hưởng nhiệt. Kết quả cho thấy rằng khi năng lượng đường tăng thì bề dày vùng ảnh hưởng nhiệt ở đáy liên kết cũng tăng theo. Khi $q_{\text {đal }}$ tăng từ $440 \mathrm{~J} / \mathrm{mm}$ lên $506 \mathrm{~J} / \mathrm{mm}$ thì độ tăng của $\mathrm{HAZ}_{1}$ tương đối chậm, còn khi $q_{\text {dl }}$ tăng từ $506 \mathrm{~J} / \mathrm{mm}$ lên $552 \mathrm{~J} / \mathrm{mm}$ thì độ tăng của $\mathrm{HAZ}_{1}$ nhanh hơn, thể hiện bởi đồ thị dốc hơn. Với $q_{\text {đl }}>552 \mathrm{~J} / \mathrm{mm}$ thì bề dày vùng ảnh hưởng nhiệt $\mathrm{HAZ}_{1}$ tăng đều theo hàm tuyến tính. 


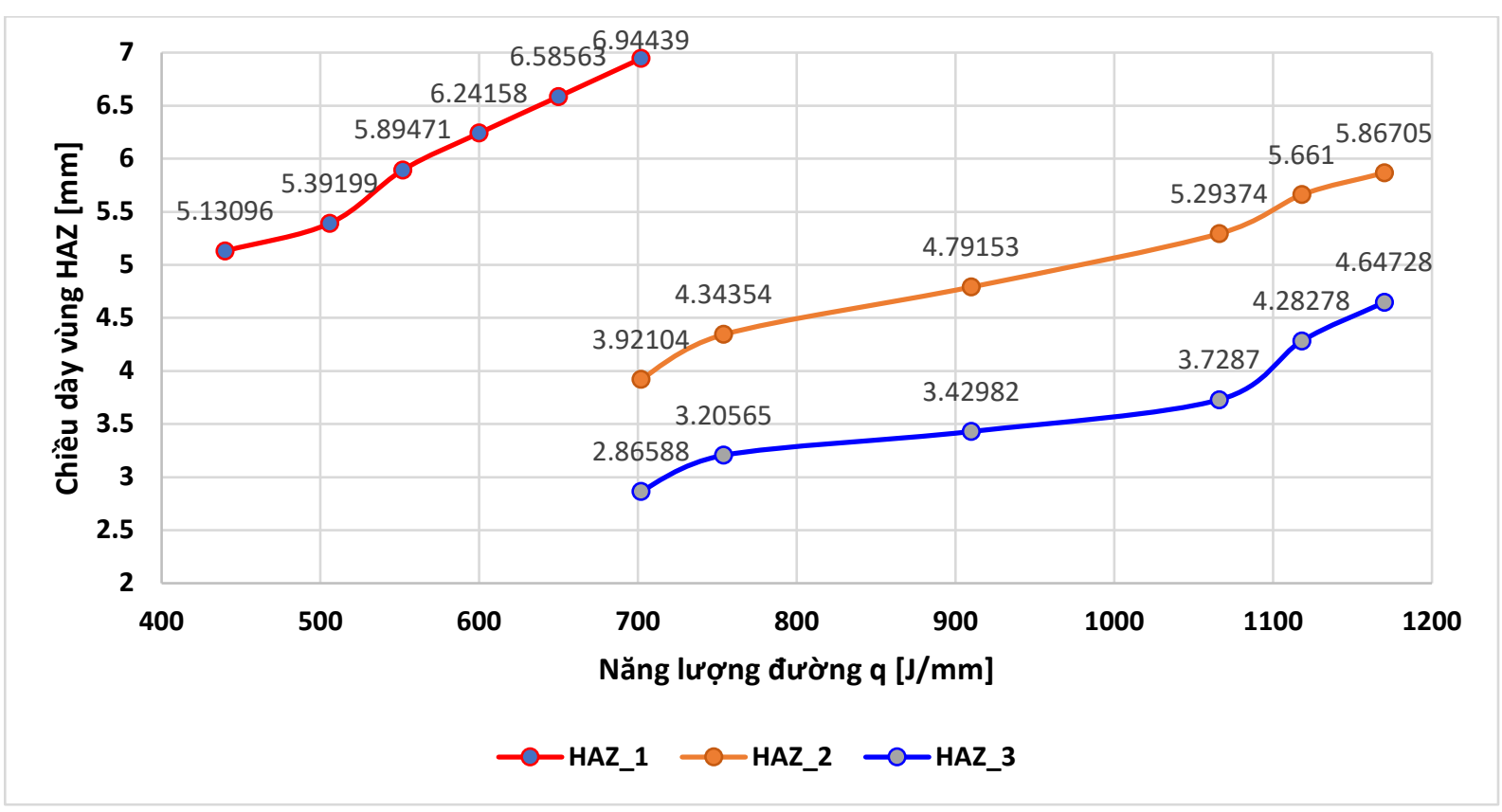

Hình 13. Ảnh hưởng của năng lượng đường đến chiều dày vùng ảnh hưởng nhiệt.

Đối với liên kết giáp mối hàn 2 lớp thì bề dày của vùng ảnh hưởng nhiệt $\mathrm{HAZ}_{2}$ và $\mathrm{HAZ}_{3}$ được quyết định bởi năng lượng đường của lớp phủ $\left(q_{\text {Ap }}\right)$ bởi vì nguồn nhiệt của lớp sau sẽ nấu chảy một phần kim loại của lớp trước và định hình lại vùng ảnh hưởng nhiệt của lớp trước [1-3].

Kết quả đo đạc kích thước của các vùng $\mathrm{HAZ}_{2}$ và $\mathrm{HAZ}_{3}$ được thống kê trong bảng 3 và cũng được biểu diễn trên đồ thị hình 13. Kết quả tính toán mô phỏng cho biết rằng chiều dày vùng ảnh hưởng nhiệt ở vị trí giữa chiều dày tấm $\left(\mathrm{HAZ}_{2}\right)$ luôn lớn hơn chiều dày vùng ảnh hưởng nhiệt ở phía trên bề mặt tấm $\left(\mathrm{HAZ}_{3}\right)$ và bé hơn chiều dày vùng ảnh hưởng nhiệt ở đáy liên kết $\left(\mathrm{HAZ}_{1}\right)$. Khi tăng năng lượng đường lớp phủ $\left(q_{đ p}\right)$ từ $702 \mathrm{~J} / \mathrm{mm}$ lên $754 \mathrm{~J} / \mathrm{mm}$ thì chiều dày vùng ảnh hưởng nhiệt $\mathrm{HAZ}_{2}$ và $\mathrm{HAZ}_{3}$ cùng tăng khá nhanh, đặc biệt là khi tăng $q_{\text {đp }}$ từ $1066 \mathrm{~J} / \mathrm{mm}$ lên $1170 \mathrm{~J} / \mathrm{mm}$ thì các vùng ảnh hưởng nhiệt $\mathrm{HAZ}_{2}$ và $\mathrm{HAZ}_{3}$ tăng đột biến. Nếu như tăng năng lượng đường lớp phủ trong dải từ $754 \mathrm{~J} / \mathrm{mm}$ đến $1066 \mathrm{~J} / \mathrm{mm}$ thì các vùng ảnh hưởng nhiệt $\mathrm{HAZ}_{2}$ và $\mathrm{HAZ}_{3}$ cùng tăng, nhưng ở mức độ chậm. Chênh lệch về độ lớn của $\mathrm{HAZ}_{2}$ khi hàn lớp phủ với năng lượng đường từ $754 \mathrm{~J} / \mathrm{mm}$ đến $1066 \mathrm{~J} / \mathrm{mm}$ chỉ là $0,9502 \mathrm{~mm}$ và chênh lệch độ lớn của $\mathrm{HAZ}_{3} \mathrm{khi}$ thay đổi năng lượng đường trong dải này chỉ là 0,52305 mm - mức tăng không đáng kể. Nói cách khác dải năng lượng đường từ $754 \mathrm{~J} / \mathrm{mm}$ đến $1066 \mathrm{~J} / \mathrm{mm}$ thích hợp để hàn lớp phủ liên kết giáp mối thép không gỉ chịu nhiệt SUS316L dày $8 \mathrm{~mm}$.

\section{Kết luận}

Bằng tính toán mô phỏng số có thể dự đoán trước được chiều dày của vùng HAZ trong liên kết hàn ở các vị trí bất kỳ, làm cơ sở cho việc chọn lựa chế độ hàn hợp lý để vừa bảo đảm liên kết đủ ngấu, vừa bảo đảm vùng ảnh hưởng nhiệt không quá lớn để tránh suy giảm độ bền của liên kết.

Nghiên cứu này đã xây dựng được cách thức xác định kích thước của vùng $\mathrm{HAZ}$ bằng mô phỏng số và tìm ra được quan hệ giữa năng lượng đường lớp lót $q_{\text {dl }}$ với chiều dày của vùng $\mathrm{HAZ}_{1}$ cũng như ảnh hưởng của năng lượng đường lớp phủ $q_{\text {dp }}$ đến chiều dày của các vùng $H A Z_{2}$ và $H A Z_{3}$ khi hàn liên kết giáp mối thép không gỉ chịu nhiệt SUS316L dày $8 \mathrm{~mm}$. Kết quả tính toán cho biết rằng khi hàn với $q_{\text {oll }}>552 \mathrm{~J} / \mathrm{mm}$ trở lên thì vùng $\mathrm{HAZ}_{1}$ tăng đều theo hàm tuyến tính và khi hàn với năng lượng đường $q_{\text {dp }}$ thuộc dải từ $754 \mathrm{~J} / \mathrm{mm}$ đến $1066 \mathrm{~J} / \mathrm{mm}$ thì các vùng $\mathrm{HAZ}_{2}$ và $\mathrm{HAZ}_{3}$ cũng tăng theo hàm gần tuyến tính và với mức tăng chậm.

Thông qua kết quả tính toán mô phỏng trường nhiệt độ phân bố trong liên kết hàn ta có thể đánh giá được khả năng hàn ngấu của liên kết và xác định được kích thước của các vùng ảnh hưởng nhiệt, để từ đó có thể biết trước được dải năng lượng đường nào là phù hợp với liên kết hàn cụ thể. Đối với liên kết giáp mối tấm thép $316 \mathrm{~L}$ dày $8 \mathrm{~mm}$ thì dải năng lượng đường phù hợp với lớp lót là $506 \mathrm{~J} / \mathrm{mm} \leq q_{d l} \leq 552 \mathrm{~J} / \mathrm{mm}$, dải năng lượng đường phù hợp với lớp phủ là $754 \mathrm{~J} / \mathrm{mm} \leq q_{\overparen{d} p} \leq 910 \mathrm{~J} / \mathrm{mm}$

\section{Lò̀i cảm ơn}

Tác giả chân thành cám ơn Trường Đại học Bách khoa Hà Nội đã cung cấp tài chính cho đề tài T2018PC-033 để nghiên cứu này thực hiện thành công.

\section{Tài liệu tham khảo}

[1] International Welding Engineer (IWE). Module 2: Materials and their behaviour during welding, International Institute of Welding (IIW). NXB GSI-SLV Duisburg, 2015. 
[2] Ngô Lê Thông, Công nghệ hàn điện nóng chảy. Tập 2 - Úng dụng, NXB Khoa học Kỹ thuật, Hà Nội, 2008.

[3] ASM handbook, Volume 6: Welding, brazing, and soldering. NXB ASM International, 1993.

[4] American Welding Society, AWS D1.6 2015 structural welding code - stainless steel. 550N.W. LeJeune Road, Miami, Florida 33126.

[5] Ngô Lê Thông, Công nghệ hàn điện nóng chảy. Tập 1 - Cơ sở lý thuyết, NXB Khoa học Kỹ thuật, Hà Nội, 2007.
[6] Radaj, D., Schweissprozess simulation grundlagen und anwendungen. Fachbuchreihe Schweisstechnik, DVS Verlag, 1999.

[7] ESI Group, Sysweld 2017 Reference manual, January 2017.

[8] J. H. Lee and Y. S. Kim, Intergranular corrosion of 316L stainless steel by aging and UNSM (Ultrasonic nano-crystal surface modification) treatment, Corrosion Science And Technology, Vol.14, No.6(2015), pp.313 324 https://doi.org/10.14773/cst.2015.14.6.313 